\title{
MicroRNA expression profiles and networks in CXCL12-stimulated human endometrial stromal cells
}

\author{
$\mathrm{JIE} \mathrm{MEI}^{1}$, MING-QING LI ${ }^{2-4}$, DA-JIN LI ${ }^{2,4}$ and HAI-XIANG SUN ${ }^{1}$ \\ ${ }^{1}$ Reproductive Medicine Center, Nanjing Drum Tower Hospital, The Affiliated Hospital of Nanjing University Medical School, \\ Nanjing, Jiangsu 210008; ${ }^{2}$ Laboratory for Reproductive Immunology, Hospital of Obstetrics and Gynecology, \\ Shanghai Medical School, Fudan University; ${ }^{3}$ Shanghai Key Laboratory of Female Reproductive Endocrine-Related Diseases, \\ Science and Technology Commission of Shanghai Municipality, Shanghai 200011; \\ ${ }^{4}$ National Population and Family Planning Commission Key Laboratory of Contraceptive Drugs \& Devices, \\ Shanghai Institute of Planned Parenthood Research, Shanghai 200032, P.R. China
}

Received October 22, 2015; Accepted November 10, 2016

DOI: $10.3892 / \mathrm{mmr} .2016 .5997$

\begin{abstract}
The chemokine stromal cell-derived factor-1 (C-X-C motif chemokine ligand 12; CXCL12) is important in the recruitment of leukocytes to the peritoneal cavity and the regulation of endometriotic tissue growth in endometriosis patients. However, the alterations in microRNA (miRNA) expression induced by CXCL12 remain to be fully elucidated. The present study evaluated key miRNAs in CXCL12-stimulated endometrial stromal cells (ESCs), and investigated the underlying cellular regulatory mechanisms of CXCL12 in endometriosis by building networks between miRNAs, genes and gene ontologies (GOs). Differential expression of miRNAs and mRNAs induced by CXCL12 stimulation in ESCs was measured using miRNA and gene chips, and it was observed that 35 miRNAs and 1,671 mRNAs were differentially expressed. Using potential target genes of the 35 miRNAs, intersections of these genes were examined and 63 intersection genes were identified. A total of 39 GOs were obtained for these intersection genes, based on information from GO databases, including immune cell chemoattractants, inflammatory and immune responses, and pathological processes of endometriotic lesions in endometriosis. In addition, miRNA-gene networks were built according to the GO and Kyoto Encyclopedia of Genes
\end{abstract}

Correspondence to: Professor Ming-Qing Li, Laboratory for Reproductive Immunology, Hospital of Obstetrics and Gynecology, Shanghai Medical School, Fudan University, 413 Zhao Zhou Road, Shanghai 200011, P.R. China

E-mail:mqli@fudan.edu.cn

Professor Hai-Xiang Sun, Reproductive Medicine Center, Nanjing Drum Tower Hospital, The Affiliated Hospital of Nanjing University Medical School, 321 Zhong Shan Road, Nanjing, Jiangsu 210008, P.R. China

E-mail: stevensunz@163.com

Key words: C-X-C motif chemokine ligand 12, endometriosis, endometrial stromal cell, microRNA, bioinformatics and Genomes databases. The present study, to the best of our knowledge, provides the most complete miRNAome and $\mathrm{mRNAome}$ profiles, and the most detailed investigation of the underlying cellular regulatory mechanisms, of the effects of CXCL12 in endometriosis. These results may facilitate the complete elucidation of the role of CXCL12 in endometriosis, and its underlying epigenetic mechanisms.

\section{Introduction}

Endometriosis affects $\sim 10 \%$ of women of reproductive age, and is a complex disease characterized by the presence of endometrial tissue outside the uterine cavity. This extra tissue subsequently leads to an ectopic growth, which results in an inflammatory response, adhesions and fibrosis, followed by chronic pelvic pain, dyspareunia and infertility (1).

Chemokines are small molecules produced during the inflammatory process and are responsible for the attraction of cells, depending on the selective receptors expressed on their membranes. Endometrial stromal cells (ESCs) exhibit the ability to invade and proliferate, and regulation of these processes is controlled by chemokines produced in the endometriotic milieu. In addition, these help recruit leukocytes to the peritoneal cavity $(2,3)$. C-X-C motif chemokine ligand 12 (CXCL12) is the single natural ligand of chemokine receptors $\mathrm{C}-\mathrm{X}-\mathrm{C}$ chemokine receptor type (CXCR) 4 and CXCR7. The refluxed endometrial cells are attracted to the peritoneal cavity via the CXCL12/CXCR4 axis (4). Overexpression of CXCR4 in stromal cells has been observed by immunohistochemistry (5). The eutopic endometrial stromal cells from endometriosis patients are attracted to the peritoneal cavity via the interaction between CXCR4 expressed on their membrane and CXCL12 produced in the peritoneal cavity. Furthermore, the activation of the CXCL12/CXCR4 axis is associated with the alternative phenotype of endometriotic cells (6). Our previous study indicated that greater levels of CXCL12 secreted from ESCs in the endometriotic milieu suppress ESC apoptosis by increasing autophagy via the nuclear factor- $\kappa \mathrm{B}$ signaling pathway (7). However, the systemic role of the CXCL12/CXCR4 axis and the molecular alterations induced 
by CXCL12 challenge in endometriosis remain to be fully elucidated.

Alterations in microRNA (miRNA) expression occur in numerous pathological and physiological processes, and miRNAs are associated with endometriosis. Dysregulation of miRNAs leads to alterations in cellular differentiation, proliferation and apoptotic processes involved in the pathogenesis of endometriosis (8). Thus, elucidating the biological consequences of dysregulated miRNAs and identifying their specific targets is necessary to understand the underlying molecular mechanisms associated with the disease.

Therefore, the present study aimed to fully elucidate the systemic role of CXCL12 in endometriosis, and investigate key miRNAs associated with CXCL12 stimulation of ESCs. Furthermore, the underlying cellular regulatory mechanisms of CXCL12 in ESCs were investigated by building networks between miRNAs, genes and gene ontologies (GOs).

\section{Materials and methods}

Subjects and sample collection. Female patients $(\mathrm{n}=20)$ of reproductive age from the Department of Obstetrics and Gynecology, Nanjing Drum Tower Hospital (Nanjing, China) were recruited as the subjects of the present study (May-June 2015). The present study was approved by the Research Ethics Committee of Nanjing Drum Tower Hospital and written informed consent was obtained from all patients prior to sampling; endometrial biopsies were obtained for research purposes only. The patients selected for inclusion in the study had not received any hormonal therapy or medication for 6 months prior to surgery and had not experienced any pelvic inflammatory-associated complications. Endometrial tissues were collected from 18 patients undergoing tubal ligation, and they were used for the control group and the CXCL12-treated group. Evaluation of endometrial histology was performed, as was a comparison of the date to the expected day of the menstrual cycle, to determine its phase. All endometrial samples used for in vitro experiments were histologically verified to be in the secretory phase of the menstrual cycle. All samples ( $>300 \mathrm{mg}$ ) were collected under sterile conditions and transported to the laboratory on ice in Dulbecco's modified Eagle's medium (DMEM)/F-12 medium (Gibco; Thermo Fisher Scientific, Inc., Waltham, MA, USA).

Cell culture and treatments. ESCs were purified and cultured from endometrial tissue as previously described (7). The ESCs exhibited $92 \%$ purity (Vimentin positive). ESCs from the same patients were used in control and treatment groups (healthy ESCs and recombinant human (rh)CXCL12-treated healthy ESCs). To clarify the miRNAome and mRNAome of CXCL12-treated ESCs, cells were treated with $100 \mathrm{ng} / \mathrm{ml}$ rhCXCL12 (R\&D Systems, Inc., Minneapolis, MN, USA) for $48 \mathrm{~h}$, whereas the control ESCs were cultured with $1 \%$ phosphate-buffered saline (PBS; Corning Incorporated, Corning, NY, USA) as the vehicle control. The rhCXCL12 treatment dose and exposure time were selected according to our previous study (7).

Isolation of total RNA. Total RNA from cultured healthy ESCs and corresponding rhCXCL12-treated healthy ESCs $(n=18)$ was extracted using Qiagen miRNeasy Mini kit (Qiagen $\mathrm{GmbH}$, Hilden, Germany). The quality of the extracted RNA was verified via absorbance measurements at wavelengths of 230, 260 and $280 \mathrm{~nm}$ using a spectrophotometer (NanoDrop 2000; Thermo Fisher Scientific, Inc.).

miRNA and mRNA microarray analysis. Total RNA was processed for miRNA and mRNA microarray analysis, using Affymetrix miRNA 4.0 and Affymetrix GeneChip Human 2.0 (Affymetrix, Inc., Santa Clara, CA, USA) as previously described (9). Following analysis, significantly differentially expressed miRNAs and mRNAs were selected according to their P-values (10).

Bioinformatics analysis. A comprehensive GO and miRNA-gene network was built to enrich the gene dataset. Genes that were differentially expressed underwent GO analysis (geneontology.org) to verify their primary function. This identification of the regulatory network of genes allowed their categorization into different hierarchies, based on their molecular function (11). Differential gene expression values and Sanger miRNA database (Gminix-Cloud Biotechnology Information, GCBI, http://www.gcbi.com.cn/gclib/html/index, Gminix Informatics Co., Ltd., Shanghai, China) interactions were used to investigate associations between miRNAs and genes, and to create an miRNA-gene network. An adjacency matrix of miRNAs and genes $A=[a i, j]$ was created according to the attributed associations between genes and miRNA, where ai, $\mathrm{j}$ represents the weight of the association between gene ai and miRNA $j$.

The regulatory status of miRNAs and genes was evaluated via GCBI. (http://www.gcbi.com.cn/gclib/html/index, Gminix Informatics Co., Ltd.). The evaluation criteria included the degrees of miRNA; the number of genes regulated by that particular miRNA, and the degree of each gene; and the number of miRNAs that regulated that particular gene. Those that exhibited the greatest degree values were identified as the most important in the analysis of the network (12).

Statistical analysis. GO analysis for all miRNA and the regulated-genes were obtained, based on the GO and Kyoto Encyclopedia of Genes and Genomes (www.genome.jp/kegg/) databases. P-values and the false discovery rate were calculated using Fisher's and $\chi^{2}$ tests (SPSS 19.0; IBM SPSS, Armonk, NY, USA), and 39 GOs were identified, with $\mathrm{P}<0.001$ considered to indicate a statistically significant difference.

\section{Results}

Microarray data. miRNAs and mRNAs induced by rhCXCL12 treatment in ESCs were measured by miRNA and gene chips. A total of 35 miRNAs and 1,671 mRNAs (Fig. 1) were differentially expressed in CXCL12-treated ESCs compared with controls.

GO analysis. The primary functions of the genes that were differentially expressed were analyzed via GO. Potential target genes of the 35 miRNAs were used to search for intersections of these miRNAs with 1,671 differentially expressed mRNAs. A total of 63 intersection genes were identified. The 39 
$\mathbf{A}$

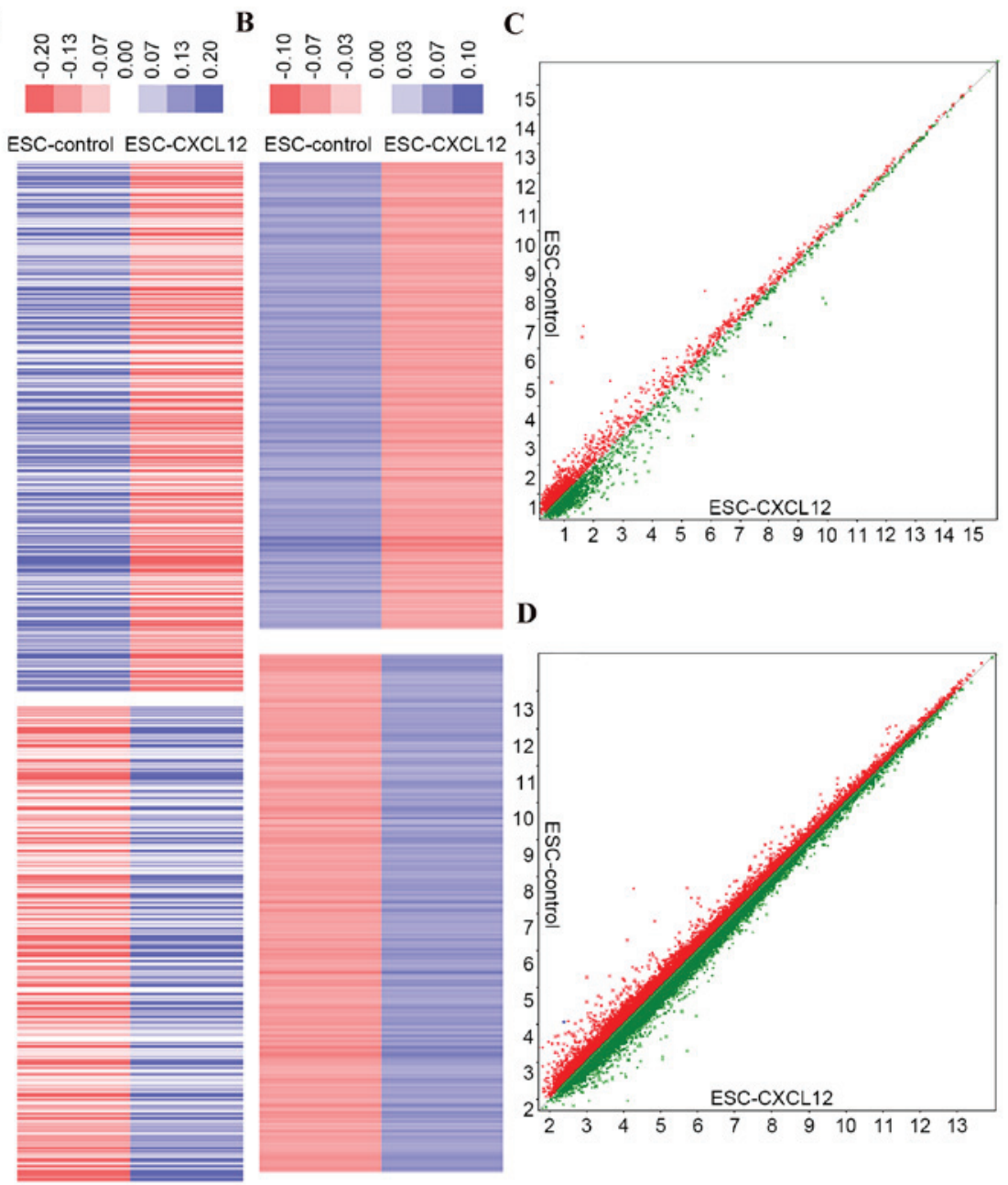

Figure 1. Microarray analysis of control and CXCL12-treated ESCs. RNA from healthy (secretory phase) and corresponding healthy ESCs treated with $100 \mathrm{ng} / \mathrm{ml}$ recombinant human CXCL12 for $48 \mathrm{~h}(\mathrm{n}=18)$ were characterized by microRNA and mRNA microarray. The heatmap indicates (A) miRNAs and (B) mRNAs with expression change fold $>2.0$. miRNAs and mRNAs that were downregulated are indicated in blue and those that were upregulated are indicated in red. A Bland-Altman plot indicates the ratio of (C) miRNA and (D) mRNA expression in healthy ESCs (vertical axis) vs. that in CXCL12-treated healthy ESCs (horizontal axis; n=18). CXCL12, C-X-C motif chemokine ligand 12; ESCs, endometrial stromal cells; miRNA, microRNA.

mRNAs that exhibited a negative association with the above 22 miRNAs were analyzed and are presented in Table I. GOs for all of the above intersection genes were obtained, based on the GO and Kyoto Encyclopedia of Genes and Genomes (www.genome.jp/kegg/) databases. P-values and the false discovery rate were calculated using Fisher's and $\chi^{2}$ tests, and 39 GOs were identified according to the standard of $\mathrm{P}<0.001$. The present study identified three classes of GOs (associated with immune cell chemoattractants, inflammatory and immune responses, and pathological processes of endometriotic lesions in endometriosis) strictly associated with CXCL12 challenge (Fig. 2), indicating that these GOs may be important in regulating the role of CXCL12 in endometriosis.

miRNA regulatory networks. Interactions between miRNAs and genes in the Sanger miRNA database led to the subsequent creation of an miRNA-gene network where significant miRNAs and genes were identified from the intersections between potential target genes of miRNAs and 1,671 differentially expressed mRNAs. A miRNA-GO network was constructed according to interactions between significant GOs and genes and associations between miRNAs and genes (Fig. 3).

\section{Discussion}

Local, peritoneal inflammation is characteristic of endometriosis, and previous studies have suggested that various components of the immune system, including chemokines, enhance the implantation of endometrial cells and the progression of the disease $(3,13)$. The CXCL12-CXCR4 axis has been demonstrated to exhibit immune functions, including lymphocyte chemotaxis and non-immune functions, including roles in tissue repair, angiogenesis and the invasion and migration involved in endometriosis $(5,14)$. Our previous study indicated that this chemokine is involved in the regulation of ESC autophagy (7). This suggested that the CXCL12-CXCR4 axis may have a broad role in the regulation of endometriosis.

Endometriotic tissue exhibits various molecular differences compared with healthy endometrial tissue, including variations in gene expression, production of proteins, responsiveness to steroids and cytokines, immune components, adhesion molecules, and proteolytic enzymes $(15,16)$. The occurrence and development of endometriosis may be based on these aberrations in molecular processes, and may be caused by as yet unknown triggers, including miRNA, which initiate these 
Table I. Differentially expressed miRNA and predicted targets.

\begin{tabular}{|c|c|c|c|c|}
\hline miRNA & Up/downregulated & Fold change & Gene ID & Up/downregulated \\
\hline hsa-miR-150-5p & Down & 0.028877827 & CCL5 & Up \\
\hline hsa-miR-4306 & Down & 0.374462719 & $\begin{array}{l}\text { ELAVL3 } \\
\text { SLA2 }\end{array}$ & Up \\
\hline hsa-miR-4286 & Down & 0.410782037 & ZFP36L1 & Up \\
\hline hsa-miR-4539 & Down & 0.416821327 & $\begin{array}{l}\text { ELAVL3 } \\
\text { HSF5 } \\
\text { RAB39B }\end{array}$ & Up \\
\hline hsa-miR-6507-5p & Down & 0.424400129 & $\begin{array}{l}\text { RGS21 } \\
\text { ZFP36L1 }\end{array}$ & Up \\
\hline hsa-miR-29b-2-5p & Down & 0.440211274 & ANKS4B & Up \\
\hline hsa-miR-18b-5p & Down & 0.440222564 & HSF5 & Up \\
\hline hsa-miR-1290 & Down & 0.44241555 & $\begin{array}{l}\text { ELAVL3 } \\
\text { EN2 }\end{array}$ & $\mathrm{Up}$ \\
\hline hsa-miR-502-5p & Down & 0.447388476 & $\begin{array}{l}\text { ELAVL3 } \\
\text { LIMD2 }\end{array}$ & Up \\
\hline hsa-miR-550a-3-5p & Down & 0.488144777 & $\begin{array}{l}\text { GP1BA } \\
\text { HOXB13 }\end{array}$ & Up \\
\hline hsa-miR-1260a & Down & 0.495246952 & $\begin{array}{l}\text { ELAVL3 } \\
\text { HOXB13 } \\
\text { LIMD2 } \\
\text { SLA2 }\end{array}$ & Up \\
\hline hsa-miR-6886-3p & Down & 0.498411064 & DPCR1 & Up \\
\hline hsa-miR-466 & Up & 2.127169505 & SORL1 & Down \\
\hline hsa-miR-4708-5p & Up & 2.223631924 & IFNG & Down \\
\hline hsa-miR-424-5p & Up & 2.348038396 & $\begin{array}{l}\text { DSEL } \\
\text { PDK4 } \\
\text { SH3BGRL2 }\end{array}$ & Down \\
\hline hsa-miR-8084 & Up & 2.398208347 & LRCH2 & Down \\
\hline hsa-miR-498 & Up & 3.346560277 & $\begin{array}{l}\text { RNASEH2B } \\
\text { SH3BGRL2 }\end{array}$ & Down \\
\hline hsa-miR-4476 & Up & 4.050657207 & FAM155A & Down \\
\hline hsa-miR-4428 & Up & 4.247547261 & SORL1 & Down \\
\hline hsa-miR-4668-5p & Up & 4.362360508 & $\mathrm{SCD}$ & Down \\
\hline hsa-miR-1184 & Up & 5.207536333 & $\begin{array}{l}\text { DSEL } \\
\text { SH3BGRL2 }\end{array}$ & Down \\
\hline hsa-miR-3613-3p & Up & 5.322665855 & $\begin{array}{l}\text { DSEL } \\
\text { LRCH2 } \\
\text { SH3BGRL2 } \\
\text { VNN2 }\end{array}$ & Down \\
\hline
\end{tabular}

A total of 22 differentially expressed miRNAs were identified in the microarray, and 39 intersection genes were predicted to have a negative association with these miRNAs. miRNA, microRNA.

molecular alterations. Certain studies have investigated the role of miRNA expression dysregulation, histone modification and DNA methylation in endometriotic cells (17). Aberrant gene expression associated with epigenetic mechanisms may induce differentiation and the subsequent appearance of an endometriotic phenotype in healthy endometrial cells $(15,16)$.

Our previous study demonstrated a greater secretion of CXCL12 in eutopic and ectopic ESCs derived from patients with endometriosis (7). The present study treated healthy
ESCs with CXCL12 to mimic the conditions in ectopic ESCs. To further elucidate the role of the CXCL12/CXCR4 axis in endometriosis, the present study detected the miRNA and miRNAome profiles in CXCL12-stimulated ESCs. The 22 miRNAs most closely associated with the CXCL12/CXCR4 axis in ESCs were reported. The present study aimed to provide the most complete miRNAome profiles, and the most detailed microRNA-gene regulatory networks described to date for the role of CXCL12 in endometriosis. The results of the present 


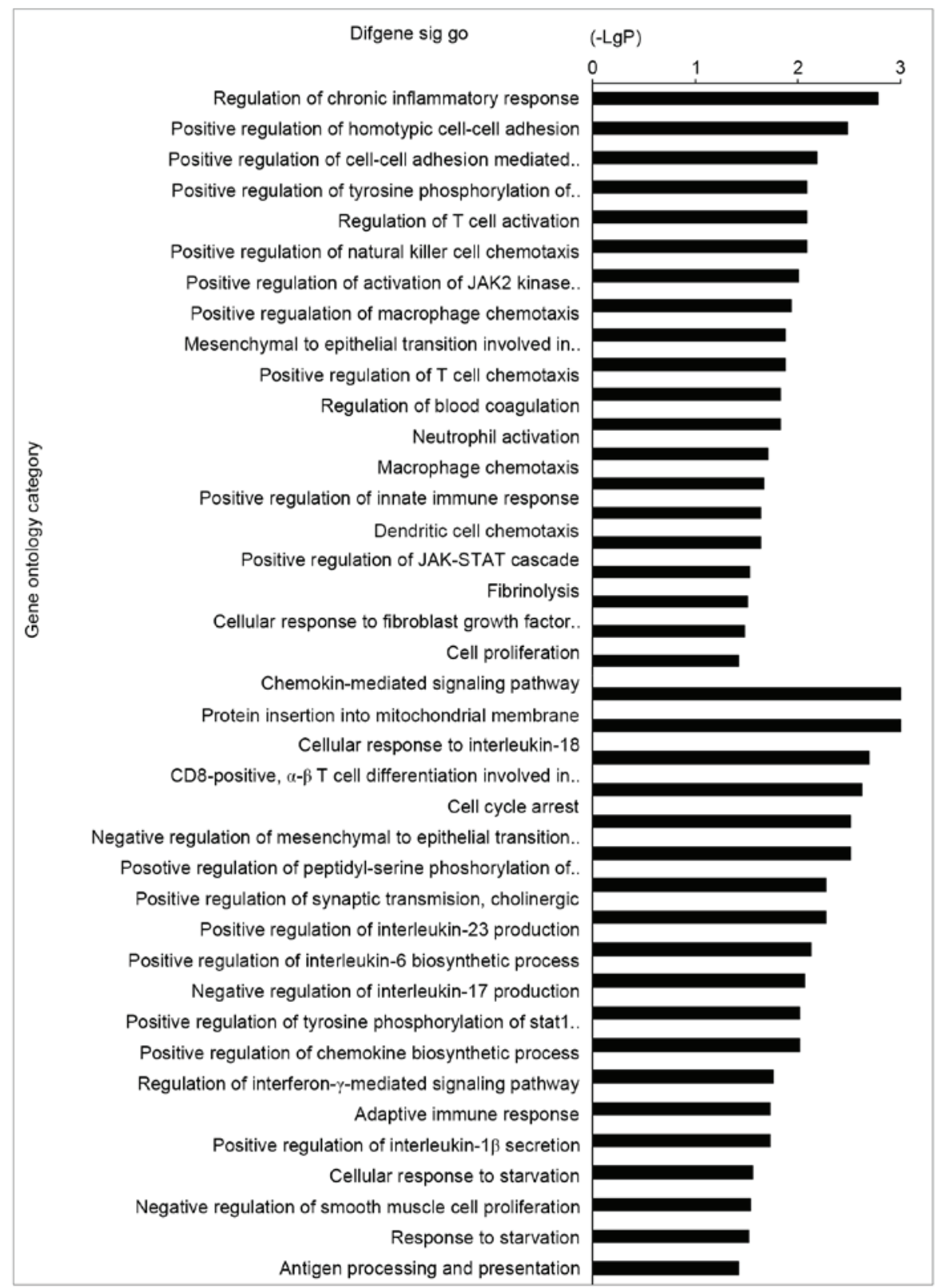

Figure 2. Histogram of significant GOs. The vertical axis represents the GO category, and the horizontal axis represents -log (P-value) of the GO; the greater the value, the greater the GO significance level. GO, gene ontology.

study provide preliminary data to advance the understanding of the pathogenesis of endometriosis.

Microarray data analysis revealed a total of 35 miRNAs and 1,671 mRNAs significantly expressed in CXCL12-treated ESCs. Bioinformatics analysis indicated that 63 mRNAs had a negative correlation with 21 differentially expressed miRNAs, and 39 significant GOs for all of the above intersection genes were obtained. GOs regarding CXCL12-challenge in ESCs were associated with: i) Chemoattractants for immune cells, including macrophages, neutrophils, natural killer (NK) cells, $\mathrm{T}$ cells and dendritic cells, and chemokine biosynthetic regulation; ii) inflammatory and immune responses, including regulation of chronic inflammatory responses, $\mathrm{T}$ cell activation, innate immune responses, antigen processing and presentation, adaptive immune responses, and interleukin production; iii) pathological processes of endometriotic lesions in endometriosis, including cell-cell adhesion, mesenchymal to epithelial transition, blood coagulation, fibrinolysis, cell proliferation, cell cycle arrest, cell-cell adhesion, Janus kinase/signal transducers and activators of transcription (STAT) signaling, and chemokine-mediated signaling.

The upregulation of CXCL12 in reflux eutopic endometrium recruits immune cells, including macrophages, NK cells, $\mathrm{T}$ cells and dendritic cells into the peritoneal cavity. These immune cells do not remove endometrial fragments in the pelvic cavity; rather an inflammatory microenvironment foundation is created, facilitating the implantation, neo-angiogenesis and proliferation of ectopic endometrial tissue (18). Conditions for the progression of the disease may include increased levels of activated peritoneal macrophages, reduced NK cell activity, an abnormal T lymphocyte response and a significantly increased number of regulatory $\mathrm{T}$ cells (19). 


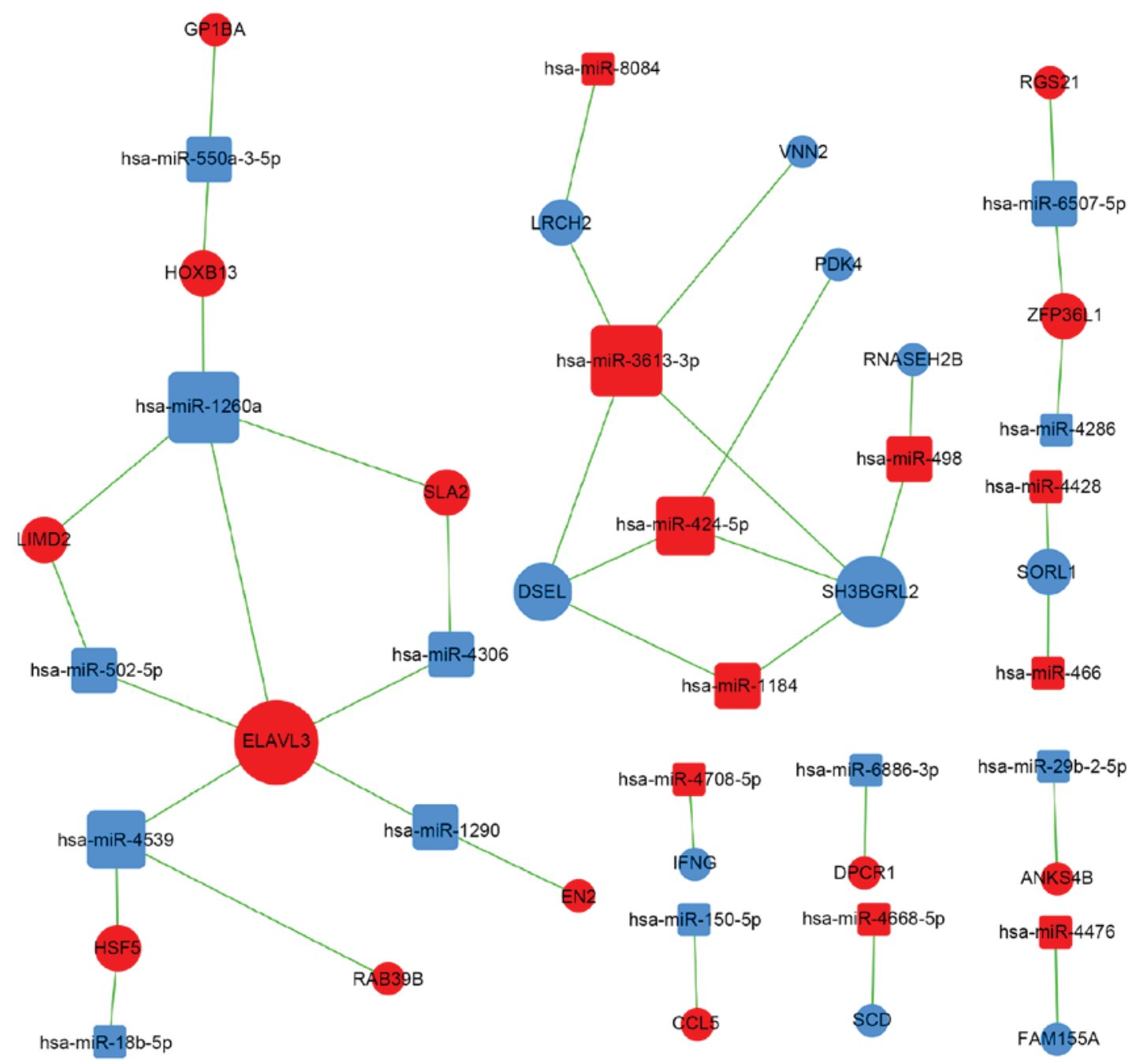

Figure 3. miRNA-GO network representing miRNA and GO interactions. Red boxes or circles denote upregulated miRNAs or mRNAs, respectively; blue boxes or circles denote downregulated miRNAs or mRNAs, respectively; and lines indicate the inhibitory effect of miRNAs on genes. The larger the area of the box or circle, the greater the degree of the miRNA or gene. GO, gene ontology; miRNA, microRNA.

CXCL12 in ESCs may directly mediate the pathological process and appearance of lesions in endometriosis, via effects on autophagy, cell-cell adhesion, mesenchymal to epithelial transition, blood coagulation, fibrinolysis and cell growth. The role of CXCL12 in ESC autophagy, which is indicated by GO analysis, is consistent with the findings of our previous study (7). The GO analysis indicated the potential role of CXCL12 in epithelial mesenchymal transition (EMT). Inflammation is regarded as an inducer of EMT in cancer progression, and EMT is considered to link inflammation and cancer (20). Epithelial cells are converted to mesenchymal cells during embryogenesis, tissue remodeling and wound healing, via EMT. The process is characterized by alterations in molecular pathways and networks; the loss of E-cadherin expression is an essential step driving this program in the initiation of human cancers (21). During arsenite-induced malignant transformation of skin cancer cells, STAT3 regulates EMT and malignant transformation (22). Although EMT occurs in endometriosis, the underlying mechanism remains to be fully elucidated. Further studies are required to identify the exact underlying mechanism of CXCL12-induced EMT in endometriosis.
miRNAs are small endogenous, single-stranded, non-coding RNA molecules that regulate the translation of specific targeted protein-coding genes (23). miRNAs regulate the expression of $50-60 \%$ of human genes without altering DNA sequences, via various epigenetic mechanisms. Numerous genes may be targeted by a single miRNA molecule and various cellular functions are induced via partial or full base-pairing with the 3'-untranslated region of the target mRNA (24).

A wide range of physiological and pathological processes have been revealed to involve miRNAs, including the cell cycle (in embryogenesis, development, differentiation and proliferation), metabolism, cell-cell communication, cell survival, apoptosis, immune responses and oncogenesis (25). Microarray studies have indicated the presence of a group of specific miRNAs that are differentially expressed between healthy endometrium without endometriosis, eutopic endometrial tissue with endometriosis and endometriotic lesions, suggesting the importance of miRNAs in the pathology of endometriosis $(26,27)$. Identification of key miRNAs via bioinformatics analysis provides information regarding an initial group of expressed miRNAs that are altered in 
CXCL12-treated ESCs and, therefore, may target genes that regulate this process. The results of the present study provide preliminary data to advance the full elucidation of the role of CXCL12 in endometriosis, and its underlying epigenetic mechanisms.

Inconclusion,themiRNAprofilesinCXCL12-overexpressed ESCs were identified in the present study using miRNA microarray analysis. The findings suggested that aberrant miRNA expression may be the epigenetic mechanism underlying the actions of CXCL12 in endometriosis, and this may provide novel and promising candidates to act as a diagnostic biomarkers and therapeutic targets of endometriosis. The present study, to the best of our knowledge, provides the most complete miRNAome profile for the evaluation of miRNAs in of CXCL12-stimulated ESCs, and the most detailed miRNA-gene regulatory network, targeting genes and GOs for investigation of the underlying miRNA mechanisms of CXCL12 in ESCs. The data from the present study will be validated in our further studies regarding endometriosis.

\section{Acknowledgements}

The present study was supported by the National Natural Science Foundation of China (grant no. 81601354), the National Science Foundation of Jiangsu Province, China (grant no. BK20160128), and the Fundamental Research Funds for the Central Universities (grant no. 021414380180) (all to J. M.), the National Natural Science Foundation of China (grant no. 81471513 and 91542108), the Development Fund of Shanghai Talents (grant no. 201557) and the Shanghai Rising-Star Program (grant no. 16QA1400800) (all to M-Q. L.).

\section{References}

1. Bulun SE: Endometriosis. N Engl J Med 360: 268-279, 2009.

2. Li MQ, Wang Y, Chang KK, Meng YH, Liu LB, Mei J, Wang Y, Wang XQ, Jin LP and Li DJ: CD4 ${ }^{+} \mathrm{Foxp}^{+}$regulatory T cell differentiation mediated by endometrial stromal cell-derived TECK promotes the growth and invasion of endometriotic lesion. Cell Death Dis 5: e1436, 2014.

3. Borrelli GM, Carvalho KI, Kallas EG, Mechsner S, Baracat EC and Abrão MS: Chemokines in the pathogenesis of endometriosis and infertility. J Reprod Immunol 98: 1-9, 2013. 2013.

4. Balkwill F: Cancer and the chemokine network. Nat Rev Cancer 4: 540-550, 2004.

5. Ruiz A, Salvo VA, Ruiz LA, Báez P, García M and Flores I: Basal and steroid hormone-regulated expression of CXCR4 in humanendometrium and endometriosis. Reprod Sci 17: 894-903, 2010

6. Leconte M, Chouzenoux S, Nicco C, Chéreau C, Arkwright S, Santulli P, Weill B, Chapron C, Dousset B and Batteux F: Role of the CXCL12-CXCR4 axis in the development of deep rectal endometriosis. J Reprod Immunol 103: 45-52, 2014.

7. Mei J, Zhu XY, Jin LP, Duan ZL, Li DJ and Li MQ: Estrogen promotes the survival of human secretory phase endometrial stromal cells via CXCL12/CXCR4 upregulation-mediated autophagy inhibition. Hum Reprod 30: 1677-1689, 2015.
8. Burney RO, Hamilton AE, Aghajanova L, Vo KC, Nezhat CN, Lessey BA and Giudice LC: MicroRNA expression profiling of eutopic secretory endometrium in women with versus without endometriosis. Mol Hum Reprod 15: 625-631, 2009.

9. Bao Y, Gao Y, Jin Y, Cong W, Pan X and Cui X: MicroRNA expression profiles and networks in mouse lung infected with H1N1 influenza virus. Mol Genet Genomics 290: 1885-1897, 2015.

10. Clarke R, Ressom HW, Wang A, Xuan J, Liu MC, Gehan EA and Wang Y: The properties of high-dimensional data spaces: Implications for exploring gene and protein expression data. Nat Rev Cancer 8: 37-49, 2008.

11. Gene Ontology Consortium: The gene ontology (GO) project in 2006. Nucleic Acids Res 34 (Database issue): D322-D326, 2006.

12. Joung JG, Hwang KB, Nam JW, Kim SJ and Zhang BT: Discovery of microRNA-mRNA modules via population-based probabilistic learning. Bioinformatics 23: 1141-1147, 2007.

13. Ramey JW and Archer DF: Peritoneal fluid: Its relevance to the development of endometriosis. Fertil Steril 60: 1-14, 1993.

14. Ahn SH, Edwards AK, Singh SS, Young SL, Lessey BA and Tayade C: IL-17A Contributes to the pathogenesis of endometriosis by triggering proinflammatory cytokines and angiogenic growth factors. J Immunol 195: 2591-2600, 2015.

15. Nasu K, Nishida M, Kawano Y, Tsuno A, Abe W, Yuge A, Takai $\mathrm{N}$ and Narahara H: Aberrant expression of apoptosis-related molecules in endometriosis: A possible mechanism underlying the pathogenesis of endometriosis. Reprod Sci 18: 206-218, 2011

16. Guo SW: Epigenetics of endometriosis. Mol Hum Reprod 15: 587-607, 2009.

17. Abe W, Nasu K, Nakada C, Kawano Y, Moriyama M and Narahara H: miR-196b targets c-myc and Bcl-2 expression, inhibits proliferation and induces apoptosis in endometriotic stromal cells. Hum Reprod 28: 750-761, 2013.

18. Barrier BF: Immunology of endometriosis. Clin Obstet Gynecol 53: 397-402, 2010.

19. Berbic M, Hey-Cunningham AJ, Ng C, Tokushige N, Ganewatta S, Markham R, Russell P and Fraser IS: The role of Foxp3+ regulatory T-cells in endometriosis: A potential controlling mechanism for a complex, chronic immunological condition. Hum Reprod 25: 900-907, 2010.

20. Zhou C, Liu J, Tang Y and Liang X: Inflammation linking EMT and cancer stem cells. Oral Oncol 48: 1068-1075, 2012.

21. Tellez CS, Juri DE, Do K, Bernauer AM, Thomas CL, Damiani LA, Tessema M, Leng S and Belinsky SA: EMT and stem cell-like properties associated with miR-205 and miR-200 epigenetic silencing are early manifestations during carcinogen-induced transformation of human lung epithelial cells. Cancer Res 71: 3087-3097, 2011.

22. Lu X, Luo F, Liu Y, Zhang A, Li J, Wang B, Xu W, Shi L, Liu X, Lu L and Liu Q: The IL-6/STAT3 pathway via miR-21 is involved in the neoplastic and metastatic properties of arsenite-transformed human keratinocytes. Toxicol Lett 237: 191-199, 2015.

23. Bartel DP: MicroRNAs: Genomics, biogenesis, mechanism, and function. Cell 116: 281-297, 2004.

24. Engels BM and Hutvagner G: Principles and effects of microRNA-mediated post-transcriptional gene regulation. Oncogene 25: 6163-6169, 2006.

25. Ohlsson Teague EM, Print CG and Hull ML: The role of microRNAs in endometriosis and associated reproductive conditions. Hum Reprod Update 16: 142-165, 2010.

26. Braza-Boïls A,Marí-Alexandre J,Gilabert J,Sánchez-Izquierdo D, España F, Estellés A and Gilabert-Estellés J: MicroRNA expression profile in endometriosis: Its relation to angiogenesis and fibrinolytic factors. Hum Reprod 29: 978-988, 2014.

27. Filigheddu N, Gregnanin I, Porporato PE, Surico D, Perego B, Galli L, Patrignani C, Graziani A and Surico N: Differential expression of microRNAs between eutopic and ectopic endometrium in ovarian endometriosis. J Biomed Biotech 2010: 369549, 2010. 